ApplicationForum

\title{
Exploration of Microbial Communities Using the Thermo Scientific Varioskan LUX Multimode Reader and the Invitrogen EVOS FL Cell Imaging System
}

Anna Hiltunen; Malena Skogman; Pia M. Vuorela \& Adyary Fallarero

Pharmaceutical Design and Discovery Group (PharmDD), Faculty of Pharmacy, University of Helsinki

Goal

This application note demonstrates the feasibility of combining the Thermo Scientific ${ }^{\mathrm{TM}}$ Varioskan $^{\mathrm{TM}}$ LUX multimode reader and Invitrogen ${ }^{\mathrm{TM}}$ EVOS $^{\mathrm{TM}}$ FL Cell Imaging System for the fluorescence-based quantification and imaging of complex biological systems, without requiring separate assay optimization.

\section{Introduction}

One of the most groundbreaking discoveries over the last 40 years has been the recognition of microbial biofilms as the predominant lifestyle of microorganisms, outside laboratory conditions. Bacterial biofilms are defined as aggregated, often sessile bacteria, which differ from free-floating cells by their slow growth and tolerance to antibiotics and immune cells. ${ }^{1}$ It is estimated that over $65 \%$ of microbial infections are biofilm-mediated, conferring biofilms a high clinical relevance. ${ }^{2}$ Moreover, in industrial settings, biofilms cause biofouling, corrosion, machinery damage and product contamination that cause delays, add cost and give rise to en-

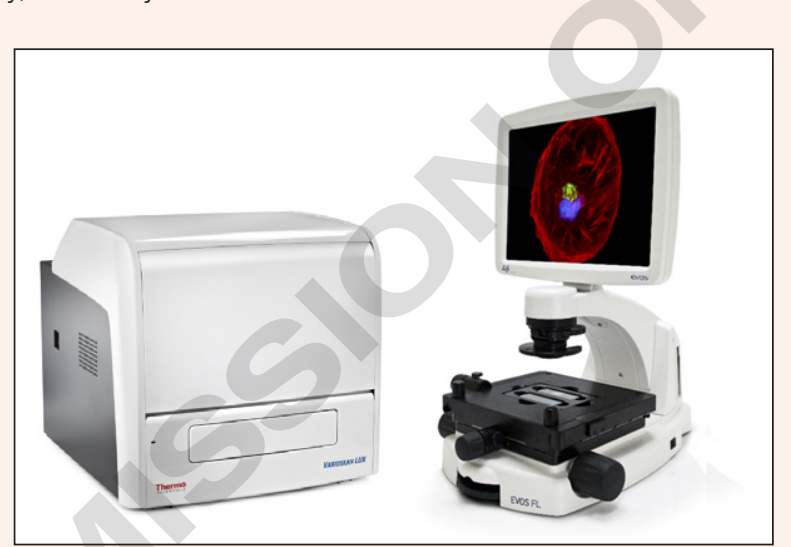

Figure 1. On the left: Varioskan LUX, a spectral scanning multimode reader, which is designed for a wide variety of microplate assays in bioscience research. On the right: EVOS FL Cell Imaging System, a fully-integrated, digital inverted instrument for four-color fluorescence and transmitted-light applications.

ergy losses. ${ }^{3}$ In a biofilm, living cells integrate into a structured multicellular community that is enclosed by a self-produced polymeric matrix, composed of polysaccharides, extracellular DNA and proteins. As biofilm cells multiply, they dynamically produce matrix components, which make the 3D-community more stable and tolerant to external threats. Thus, the study of the biofilm matrix provides essential clues into the community's functional behavior, which are critical for the understanding of biofilms from an industrial, environmental, biomedical, pharmaceutical and even purely microbiological perspective.

In this application note, we describe experiments conducted to evaluate the use of the Varioskan LUX multimode reader in conjunction with the EVOS FL Cellular Imaging System for quantification and visualization of the biofilm matrix, respectively.

The high quality output data, combined with the convenience and user-friendliness of both instruments, make them excellent tools for simultaneous quantification and imaging of fluorescent signals in complex (multi-component) biological systems, without the need for investments in more costly High Content Screening (HCS) systems. Moreover, assay conditions that were found to be optimal for Varioskan LUX-based signal quantification were directly applicable to EVOS FL-based imaging.

\section{Materials and Methods}

\section{Instruments}

- Varioskan LUX multimode reader

(Thermo Fisher Scientific, VL0000D0-VLBLATD2)

- EVOS FL Cell Imaging System

(Thermo Fisher Scientific, AMF4300)

\section{Bacterial strains, reagents and materials}

- Staphylococcus aureus ATCC 25923

- Staphylococcus epidermidis RP62A

-Wheat germ agglutinin, Alexa Fluor ${ }^{\mathrm{TM}} 488$ conjugate, WGA (Thermo Fisher Scientific, W11261)

- Tryptic Soy Broth, TSB (Sigma-Aldrich $\left.{ }^{\oplus}, 22092\right)$

- Lonza BioWhittakerTM Phosphate Buffer Saline,

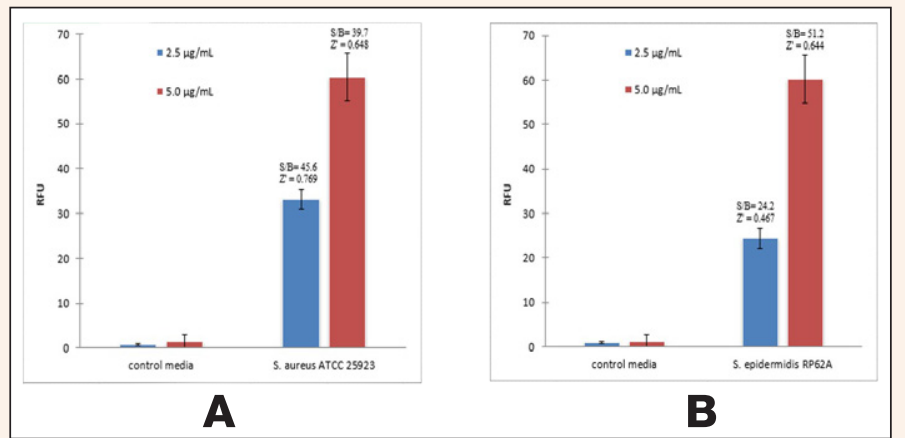

Figure 2. WGA-Alexa Fluor 488 fluorescent conjugate-based quantification of PNAG in the biofilm matrix of $S$. aureus $(A)$ and $S$. epidermidis (B) strains, measured using Varioskan LUX multimode reader. 
PBS (BioWhittaker, BE17-516F)

- Thermo Scientific ${ }^{\mathrm{TM}}$ Nunc ${ }^{\mathrm{TM}}$ 96-microtiter well plates with Nunclon ${ }^{\mathrm{TM}}$ Delta surface (Thermo Fisher Scientific, 163320)

- Borosilicate glass coupons (BioSurface Technologies

Corporation, RD128-GL)

\section{Test set-up}

S. aureus and S. epidermidis biofilms were grown for 18 hours at $37^{\circ} \mathrm{C}, 200 \mathrm{rpm}$ in TSB, in microtiter well plates or in borosilicate glass coupons (diameter 1.27 $\mathrm{cm}$, height $0.4 \mathrm{~cm}$ ). For quantification of the matrix, a wheat germ agglutinin-Alexa Fluor 488 fluorescent conjugate (WGA) was used, as it binds to poly- $\mathrm{N}$-acetylglucosamine (PNAG) residues present as one component of the biofilm matrix. Biofilm formed in microtiter well plates were washed with sterile PBS, then $125 \mu$ l of WGA was added and biofilms were incubated in the dark for 2 hours at $+4^{\circ} \mathrm{C}$. After removing the unbound dye, samples were washed again (3 times) with sterile PBS and left to air-dry for 15 minutes at RT prior to the addition of $125 \mu \mathrm{l}$ of 33\% acetic acid.

Biofilms were recovered by physically scrapping off the attached bacteria on the bottom of the wells. The resulting sample was incubated at $+37^{\circ} \mathrm{C}$ for 1 hour and sonicated for 30 seconds, before measuring the WGA fluorescence $(\lambda$ exc $=495$ $\mathrm{nm} ; \mathrm{em}=520 \mathrm{~nm}$ ) using Varioskan LUX (top reading function). For fluorescence imaging, $S$. aureus biofilms formed in glass coupons were incubated with WGA as described earlier. Immediately after the 2 hour incubation step, the unbound dye was removed and images of the stained biofilms were taken directly with the EVOS FL microscope using the GFP light cube ( $\lambda$ exc $=470 / 22 \mathrm{~nm} ; \lambda e m=510 / 42 \mathrm{~nm})$. The amount of biofilms present in the bottom surface of four wells in a 96-microtiter well plate was found to correspond to those formed in one borosilicate glass coupon.

\section{Results and Discussion}

Two concentrations of the WGA probe were evaluated to optimize the PNAG fluorescence-based quantification of the matrix of biofilms formed by two staphylococcal strains (Figure 2). This optimization was substantially simplified due to the fact that Varioskan LUX has an automatic dynamic range feature that allows optimal fluorescence intensity measurements for a wide range of registered signals within one plate. This is particularly significant, in that laborious, multi-step protocols require moderately expensive fluorescence probes. The highest fluorophore concentration ( $5 \mu \mathrm{g} / \mathrm{ml}$, red bars) has been utilized in earlier studies ${ }^{4,5}$. However, a lower concentration ( $2.5 \mu \mathrm{g} / \mathrm{ml}$, blue bars) was also found to result in a high screening window coefficient and signal-to-background values ( $Z^{\prime}>0.46$ and $\mathrm{S} / \mathrm{B}>24.2$, respectively) for both staphylococcal strains.

To further study the spatial distribution of the biofilm matrix, S. aureus samples stained with the same fluorescent-based protocol were visualized with an EVOS FL Cell Imaging System (Figure 3). A network-like organization of the PNAG around biofilm cells was clearly visible, even when using the lower fluorophore concentrations. In conjunction with the results of Figure 2, these data indicate that PNAG is predominant in the S. aureus ATCC 25923 biofilm matrix. It appears to be homogenously distributed around the cells and not only occupying specific regions around the microbial community. The PNAG network was less structured towards the edge of the coupons, thus re-affirming the necessity of taking edge effects into account. Of note, no modifications of the staining protocol used for quantification had to be done prior to imaging, confirming the convenience of utilizing the Varioskan LUX multimode reader and EVOS FL Cellular Imaging System in tandem for a more complete characterization of microbial communities, and likely other similarly complex biological systems.

\section{References}

1. M. Alhede, K.N. Kragh, K. Qvortrup, M. Allesen-Holm, M. van Gennip, L.D. Christensen, P. Jensen, A.K. Nielsen, M. Parsek, D. Wozniak, S. Molin, T. TolkerNielsen, N. Høiby, M. Givskov, T. Bjarnsholt, Phenotypes of non-attached Pseudomonas aeruginosa aggregates resemble surface attached biofilm, PLoS One, 6 (2011) e27943.

2. M. Otto, Staphylococcal infections: Mechanisms of biofilm maturation and detachment as critical determinants of pathogenicity, Annu Rev Med, 64 (2013) $175-188$.

3. R.M. Donlan, J.W. Costerton, Biofilms: survival mechanisms of clinically relevant microorganisms., Clin Microbiol Rev, 15 (2002) $167-193$.

4. M.E. Skogman, P.M. Vuorela, A. Fallarero, Combining biofilm matrix measurements with biomass and viability assays in susceptibility assessments of antimicrobials against Staphylococcus aureus biofilms, J Antibiot (Tokyo), 65 (2012) 453-459.

5. E. Burton, N. Yakandawala, K. LoVetri, M. Madhyastha, A microplate spectrofluorometric assay for bacterial biofilms., J Ind Microbiol Biotechnol, 34 (2007) 1-4. 